4 Aya Tanaka ${ }^{1,4}$, Michiko Matsuse ${ }^{1}$, Vladimir Saenko ${ }^{2}$, Tomoe Nakao ${ }^{1,5}$, Kosho

5 Yamanouchi $^{6}$, Chika Sakimura ${ }^{6}$, Hiroshi Yano ${ }^{4}$, Eijun Nishihara ${ }^{7}$, Mitsuyoshi Hirokawa ${ }^{8}$,

6 Keiji Suzuki ${ }^{1}$, Akira Miyauchi ${ }^{9}$, Susumu Eguchi ${ }^{6}$, Ko-ichiro Yoshiura ${ }^{3}$, Shunichi

7 Yamashita $^{1}$, Takeshi Nagayasu ${ }^{4}$, Norisato Mitsutake ${ }^{1}$

8

9

\section{TERT mRNA expression as a novel prognostic marker in papillary thyroid} carcinomas

${ }^{1}$ Department of Radiation Medical Sciences, ${ }^{2}$ Department of Radiation Molecular Epidemiology, ${ }^{3}$ Department of Human Genetics, Atomic Bomb Disease Institute, Nagasaki University, Nagasaki, Japan.

${ }^{4}$ Department of Surgical Oncology, ${ }^{5}$ Department of Endocrinology and Metabolism, ${ }^{6}$ Department of Surgery, Nagasaki University Graduate School of Biomedical Sciences, Nagasaki, Japan.

${ }^{7}$ Department of Internal Medicine, ${ }^{8}$ Department of Diagnostic Pathology and Cytology, ${ }^{9}$ Department of Surgery, Kuma Hospital, Kobe, Japan.

Aya Tanaka, MD

Department of Radiation Medical Sciences, Atomic Bomb Disease Institute, Nagasaki University

1-12-4 Sakamoto, Nagasaki 852-8523, Japan

Department of Surgical Oncology, Nagasaki University Graduate School of Biomedical Sciences

5 1-7-1 Sakamoto, Nagasaki 852-8501, Japan

26 a_matsumo812@yahoo.co.jp 
Michiko Matsuse, $\mathrm{PhD}$

29 Department of Radiation Medical Sciences, Atomic Bomb Disease Institute, Nagasaki

30 University. 1-12-4 Sakamoto, Nagasaki 852-8523, Japan.

31 michikom@nagasaki-u.ac.jp

33 Vladimir Saenko, $\mathrm{PhD}$

34 Department of Radiation Molecular Epidemiology, Atomic Bomb Disease Institute, 35 Nagasaki University.

36 1-12-4 Sakamoto, Nagasaki 852-8523, Japan.

37 saenko@nagasaki-u.ac.jp

Tomoe Nakao, MD

40

Department of Radiation Medical Sciences, Atomic Bomb Disease Institute, Nagasaki

41 University

42

1-12-4 Sakamoto, Nagasaki 852-8523, Japan

Department of Endocrinology and Metabolism, Nagasaki University Graduate School of

$44 \quad$ Biomedical Sciences

1-7-1 Sakamoto, Nagasaki 852-8501, Japan

46 tomoenakao@gmail.com

47

Kosho Yamanouchi, MD PhD

49 Department of Surgery, Nagasaki University Graduate School of Biomedical Sciences

50 1-7-1 Sakamoto, Nagasaki 852-8501, Japan

51 ymanouch@gk9.so-net.ne.jp

53 Chika Sakimura, MD PhD

54 Department of Surgery, Nagasaki University Graduate School of Biomedical Sciences 
56 csaki@nagasaki-u.ac.jp

Hiroshi Yano, MD PhD

59 Department of Surgical Oncology, Nagasaki University Graduate School of Biomedical

60 Sciences

61 1-7-1 Sakamoto, Nagasaki 852-8501, Japan

62 hiroyano@nagasaki-u.ac.jp

63

64 Eijun Nishihara, MD PhD

65 Department of Internal Medicine, Kuma Hospital

66

8-2-35 Shimoyamate-dori, Chuo-ku, Kobe 650-0011, Japan.

67 nishihara@kuma-h.or.jp

68

69

Mitsuyoshi Hirokawa, MD PhD

70

Department of Diagnostic Pathology, Kuma Hospital

8-2-35 Shimoyamate-dori, Chuo-ku, Kobe 650-0011, Japan.

mhirokawa@kuma-h.or.jp

Keiji Suzuki, PhD

Department of Radiation Medical Sciences, Atomic Bomb Disease Institute, Nagasaki

76 University. 1-12-4 Sakamoto, Nagasaki 852-8523, Japan.

kzsuzuki@nagasaki-u.ac.jp

79 Akira Miyauchi, MD PhD

80 Department of Surgery, Kuma Hospital

8-2-35 Shimoyamate-dori, Chuo-ku, Kobe 650-0011, Japan. 
miyauchi@kuma-h.or.jp

83

84 Susumu Eguchi, MD PhD

85

Department of Surgery, Nagasaki University Graduate School of Biomedical Sciences

86 1-7-1 Sakamoto, Nagasaki 852-8501, Japan

87 sueguchi@nagasaki-u.ac.jp

88

89 Ko-ichiro Yoshiura, MD PhD

90 Department of Human Genetics, Atomic Bomb Disease Institute, Nagasaki University

91 1-12-4 Sakamoto, Nagasaki 852-8523, Japan.

92 kyoshi@nagasaki-u.ac.jp

93

94 Shunichi Yamashita, MD PhD

95 Department of Radiation Medical Sciences, Atomic Bomb Disease Institute, Nagasaki

96 University. 1-12-4 Sakamoto, Nagasaki 852-8523, Japan.

97 shun@nagasaki-u.ac.jp

98

99

Takeshi Nagayasu, MD PhD

100 Department of Surgical Oncology, Nagasaki University Graduate School of Biomedical

101 Sciences

102 1-7-1 Sakamoto, Nagasaki 852-8501, Japan

103 nagayasu@nagasaki-u.ac.jp

104

105

106 Running title: TERT expression as a prognostic marker in PTC

107

108 
109 Key words: TERT, TERT promoter mutation, TERT expression, prognostic marker, 110 papillary thyroid carcinoma

111

112

113 Correspondence and reprint requests: Norisato Mitsutake, $\mathrm{MD}, \mathrm{PhD}$.

114 Department of Radiation Medical Sciences, Atomic Bomb Disease Institute, Nagasaki

115 University, Nagasaki, Japan.

116 1-12-4 Sakamoto, Nagasaki 852-8523, Japan.

117 Tel: $+81-95-819-7116$

118 Fax: $+81-95-819-7117$

119 E-mail: mitsu@nagasaki-u.ac.jp

120 
ABSTRACT

122 Background: TERT promoter mutations have been found in a subset of papillary thyroid carcinomas (PTCs) and are associated with tumor aggressiveness and worse prognosis. However, little is known about the status of TERT mRNA expression and its relation to TERT promoter mutations and clinicopathological features.

Methods: We analyzed 159 PTC samples for TERT promoter mutations using direct DNA sequencing. TERT expression was measured using quantitative RT-PCR. To examine low allelic frequency of TERT promoter mutations with high sensitivity, we used droplet digital PCR (ddPCR). The relationship between the status of the TERT promoter mutation/expression and clinicopathological features including recurrence risk was statistically analyzed.

Results: TERT promoter mutations were found in 20 cases (12.6\%). However, TERT expression was observed not only in the mutation-positive tumors but also in 56 of 139 (40.3\%) mutation-negative tumors. Among them, we detected low allelic frequency of TERT promoter mutations in three samples (5.4\%) using ddPCR. We confirmed a significant association between TERT promoter mutations and aggressive clinicopathological features in this series. The risk of recurrence of TERT mutationnegative/expression-positive tumors was significantly higher than that of the mutationnegative/expression-negative tumors, suggesting that TERT expression even in absence of a mutation confers a negative influence on PTCs. Moreover, when we reclassified the mutation-negative cases into two groups based on the TERT expression levels: expression-negative/expression levels below the 80th percentile and expression levels above the 80th percentile because minimal expression may have a negligible clinical impact, a higher hazard ratio for recurrence was observed. Interestingly, TERT expression levels in the mutation-negative PTCs were inversely correlated with patient age and the presence of BRAF mutations.

Conclusions: We confirm a strong correlation between the presence of TERT promoter 
148 mutations and aggressive clinicopathological features in this PTC series. In addition,

149 there were PTCs showing high TERT mRNA expression even in the absence of TERT

150 promoter mutations. These cases also showed a significantly higher recurrence rate.

151 Since the TERT promoter mutations are observed only in elderly patients, TERT mRNA

152 expression can be a useful prognostic marker especially in younger PTC patients.

153

154

155 


\section{INTRODUCTION}

157 The incidence of papillary thyroid carcinoma (PTC) has been increasing worldwide (1).

158 PTC has generally a favorable prognosis; however, approximately $10-15 \%$ of patients have recurrences either locally or/and at distant sites, some of which become refractory to treatment $(2,3)$. To distinguish between high-risk and low-risk cases, there have been many studies evaluating the value of molecular markers to predict PTC aggressiveness and prognosis.

The $B R A F^{V 600 E}$ mutation is the most frequent genetic change in PTC. Its prevalence varies from 30 to $80 \%$ (4), probably depending on the population. Many studies have indicated an association between the presence of the $B R A F^{V 600 E}$ mutation and aggressive clinicopathological features; however, its prognostic value, especially as an independent marker, is still debated. According to our results, the mutation rate in Japan is high ( $\sim 80 \%)$, and we did not find any correlation between the presence of the $B R A F^{V 600 E}$ mutation and aggressive clinicopathological features and worse prognosis in our series (5).

Recently, mutations in the promoter region of the telomerase reverse transcriptase (TERT) gene have been found in many types of cancers including thyroid carcinomas. There are two hot spots, called C250T (chr5: 1,295,250C > T) and C228T (chr5: 1,295,228C > T) (6, 7), and these are mutually exclusive. The average rate of the presence of these mutations in PTC has been reported to be around $10 \%(8)$, and there seems to be no major differences between populations. The mutations create a binding motif for the E26 transformation-specific (ETS) transcription factors and upregulate TERT mRNA expression, especially when the ETS family members are activated (e.g. by BRAF ${ }^{\mathrm{V} 600 \mathrm{E}}$ ) (9-11). While the primary function of TERT is to maintain telomere length, there is increasing evidence regarding its telomerase-independent oncogenic functions through NF- $\kappa \mathrm{B}, \mathrm{Wnt} / \beta$-catenin, and MYC pathways (12-14). In PTC, many studies have 
demonstrated that the co-existence of the $B R A F^{V 600 E}$ mutation and TERT promoter mutations is strongly associated with aggressive features and worse prognosis $(5,15-27)$. Moreover, TERT promoter mutations seem to be also associated with anaplastic transformation (28).

However, regarding the impact of TERT promoter mutations, two recent findings should be considered. First, Paulsson et al. have reported that there is a subset of cases showing TERT mRNA expression in the absence of TERT promoter mutations in follicular thyroid tumors (adenomas, follicular tumors with uncertain malignant potential, and carcinomas) (29). In that study, the authors have also demonstrated that tumors with positive TERT mRNA expression showed a shorter time to recurrence compared with TERT expressionnegative carcinomas (29). However, many of the TERT-expressing tumors harbored TERT promoter mutations, and the prognostic value of TERT mRNA expression independent of the presence of TERT promoter mutations has not been analyzed. Muzza et al. also demonstrated TERT protein expression in PTCs without TERT promoter mutations (30). However, the number of analyzed cases was limited, and a correlation analysis between presence of TERT expression and clinicopathological findings was not presented (30). Secondly, according to The Cancer Genome Atlas (TCGA) data, TERT promoter mutations were not clonal in PTCs (31). The mutant allele frequency varied from $5 \%$ to $50 \%$ (average $23 \%$ ) and there were cases with low allelic frequency that may not be detectable by regular Sanger sequencing (31).

In PTC, little is known about the relationship between TERT mRNA expression and clinicopathological features. Therefore, we measured TERT mRNA expression levels and allelic frequency of the TERT promoter mutations in PTCs in the present study, and analyzed their impact on clinicopathological features, especially as a prognostic marker. 


\section{MATERIALS AND METHODS}

\section{1 PTC samples and patient information}

212 We collected 159 PTC samples operated between November 2001 and December 2017 at 213 Nagasaki University Hospital (Nagasaki, Japan) and Kuma Hospital (Kobe, Japan).

214 Clinicopathological data were collected from the patients' medical records. Patient age at 215 operation ranged 14-81 years old (median age: 54 years old, 17.0\% male). For staging, 216 the AJCC/TNM staging system (8th edition) was used $(32,33)$. The histological subtypes 217 were: 146 classic PTCs (25 were microcarcinomas), 10 follicular variant of PTCs (four 218 were microcarcinomas), two diffuse sclerosing variant of PTCs, and one tall cell variant 219 of PTC. The study protocol was approved by the institutional review boards of Nagasaki 220 University and Kuma Hospital. Written informed consent was obtained from each patient. 221 Fresh tumor tissue samples were obtained during surgical operations, snap-frozen in liquid nitrogen, and stored at $-80^{\circ} \mathrm{C}$. DNA and total RNA were extracted at the same time using ISOGEN reagent (Nippon Gene) according to the manufacturer's protocol.

\section{Direct DNA sequencing}

The mutational status of BRAF (around V600) and the promoter region of TERT were analyzed by direct DNA sequencing (the Sanger method) as described previously (5).

\section{Quantitative reverse transcription polymerase chain reaction (qRT-PCR)}

Total RNA was reverse transcribed using a High Capacity RNA-to-cDNA kit (Applied Biosystems). The following PCR reactions were done using SYBR Premix Ex TaqII (TaKaRa Bio) in a Thermal Cycler Dice real-time system (TaKaRa Bio). The cycle threshold (CT) value, which was determined using the second derivative method, was used to calculate relative expression. The TERT mRNA levels were normalized using TATA-binding protein (TBP) mRNA expression as a reference. Primer sequences are: TERT ex6-7 F, 5'-AGCCACGTCTCTACCTTGAC-3' and TERT ex7-8 R, 5'- 

and TBP ex3 R, 5'-TGGTGTTCTGAATAGGCTGTGG-3'.

\section{Droplet digital PCR (ddPCR)} ddPCR was performed using ddPCR Supermix for Probes (Bio-Rad Laboratories) in a QX100 droplet generator (Bio-Rad Laboratories), a C1000 Touch thermal cycler (BioRad Laboratories), and a QX100 droplet reader (Bio-Rad Laboratories). Probes used for the ddPCR were: TERT mut, 5'-/56-FAM/C+CC+C+T+TC+CGG/3IABkFQ/-3', and

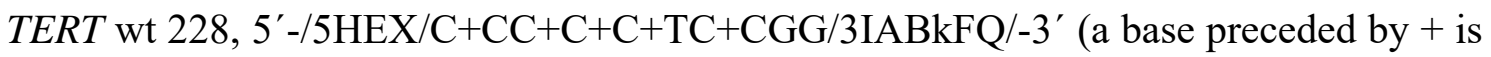
Locked Nucleic Acid). Primers were same as those used in direct DNA sequencing. It is possible to discriminate between $\mathrm{C} 228 \mathrm{~T}$ and C250T in the two-dimensional (2D) display using above two probes at the same time because the TERT mut probe can bind to both $\mathrm{C} 228 \mathrm{~T}$ and C250T, but the wt probe can bind to only C228. In the case of the C250T mutation, both FAM and HEX signals are detected, while only a FAM signal is detected when the mutation is $\mathrm{C} 228 \mathrm{~T}$.

\section{Recurrence as an endpoint}

Disease recurrence was defined as a surgically removed and pathologically verified local lesion or regional metastasis/distant metastasis detected by ultrasound, scintigraphy, or other imaging not earlier than six months after initial treatment. The time to recurrence was calculated based on the date of reoperation or on the date of recurrence detection by medical imaging.

\section{Statistical Analysis}

261 Univariate Fisher's or Fisher-Freeman-Halton exact tests were used for categorical data. Pairwise statistical comparisons of proportions in more than two groups were performed with the COMPPROP macros in SAS (http://www2.sas.com/proceedings/sugi31/204- 
265 Critchlow-Fligner multiple comparison procedure for continuous data were used to compare characteristics in different PTC subgroups. To analyze recurrence-free survival (RFS), the Kaplan-Meier method and log-rank test were used. Factors affecting RFS were assessed in Cox proportional hazard models. To determine a threshold of the TERT expression level based on the concept that minimal expression compared with relatively higher expression by the TERT promoter mutations could have negligible effect on clinical behavior, we first calculated hazard ratios (HRs) in serial optimal Cox models for each cut-off percentile (mRNA expression-negative plus expression levels below each percentile cases vs expression levels above the percentile cases) in five percentile increment. We next ran Kaplan-Meier analyses using percentiles which demonstrated relatively higher HR. Then optimal threshold was selected based on the log-rank p-value. Multivariable logistic regression models were used to identify factors associated with extrathyroidal extension or pT category. Analyses with very small numbers of outcomes $(<5$ per cell) or when quasi-complete separation was observed were conducted using Firth's approach to bias-reducing penalized maximum likelihood fit. Non-automatic model optimization was routinely performed using the Akaike information criteria. Stepwise variable selection was applied to the models amendable to automatic optimization. Once the most appropriate model was determined, the maximum likelihood estimates of the respective parameters and their Wald-type 95\% confidence intervals were calculated. Statistical assessments were performed using the 3.71 release of SAS Studio for the 9.4M5 version of SAS (SAS Institute) or IBM SPSS Statistics Version 24 software (IBM). Graphs were drawn using GraphPad Prism 6 (GraphPad). All p-values were 2sided and considered significant if $\mathrm{p}<0.05$. 
292 We first screened 159 PTC samples for mutations of the TERT promoter region (C228T 293 and C250T) by direct DNA sequencing. TERT promoter mutations were found in 20 294 (12.6\%) samples, all of which were C228T, and there was no C250T substitution in the 295 current series. We next examined TERT mRNA expression by real-time qRT-PCR. TERT expression was confirmed in all of the TERT promoter mutation-positive samples. Interestingly, even among 139 mutation-negative samples, 56 (40.3\%) showed TERT expression. We then explored the possibility that there are tumors with low allelic frequency of TERT promoter mutations that are not detectable by regular sequencing. To investigate the presence of the mutations with high sensitivity, we used ddPCR. First, the detection limit of the ddPCR for the two TERT promoter mutations was determined using serial dilutions of the PCR product of the TERT promoter region containing C228T or C250T in the PCR product of the wild-type promoter. In our hands, the detection limit of the mutant allele frequency was approximately $0.25 \%$ (Supplementary Fig. S1). We then analyzed all of the 56 TERT-expressing samples using ddPCR. We identified the mutation with low allelic frequency in three samples (5.4\%), hereafter PTC A, B, and C (Fig. 1A). According to the 2D display, all harbored a C228T mutation. The allelic frequencies of the mutant were $17 \%, 10 \%$, and $5 \%$ in PTC A, B, and C, respectively (Supplementary Table S1). Since tumor tissues consist not only of tumor cells but also of stromal, endothelial, and blood cells, the allelic frequency of the mutant in the tumor cells was corrected for tumor purity based on the ratio of the TERT promoter mutation to the $B R A F^{V 600 E}$ mutation because the $B R A F^{V 600 E}$ mutation is considered a clonal monoallelic mutation in all tumor cells according to the data obtained using next-generation sequencing and immunohistochemistry $(34,35)$. However, one should note that there is also some debate about the allelic frequency of the $B R A F^{V 600 E}$ mutation. Guerra et al.

316 reported variable BRAF mutant allelic frequencies in PTCs using pyrosequencing (36).

317 For the present study, we assumed that all tumor cells were $B R A F^{V 600 E}$ positive. After the 
correction, the allelic frequencies of the TERT promoter mutation in tumor cells were as

319 follows: PTC A, 14\%; PTC B, 4\%; and PTC C, 3\%, which means that 28\%, 8\%, and 6\%

320 of the tumor cells had the TERT promoter mutation in PTC A, B, and C, respectively

321 (Supplementary Table S1). We then retrospectively checked the chromatograms of the direct DNA sequencing results of these samples. There were discrete peaks of C228T (Fig. 1B), but it was impossible to confidently discriminate these signals from background signals. The results of direct DNA sequencing, expression analysis, and ddPCR are summarized in Fig. 2A-C. Taken together, TERT expression was observed in all of the tumors with the TERT promoter mutation as expected, even in the cases with low allelic frequency; however, TERT expression was also detected in $38 \%$ of mutation-negative cases, suggesting that there are other mechanisms to upregulate TERT expression.

Relationship between TERT mutational/expression status and clinicopathological features

We analyzed the relationship between the status of the TERT promoter mutation/expression and clinicopathological features. We classified the 159 cases into three groups: the TERT promoter mutation-negative/mRNA expression-negative group (mut-/exp-), the TERT promoter mutation-negative/mRNA expression-positive group (mut-/exp+), and the TERT promoter mutation-positive group (mut+/exp+). As shown in Table 1, tumors with the mutation $\left(\operatorname{mut}+/ \exp ^{+}\right)$showed statistically significant differences in age, extrathyroidal extension, stage II/III/IV, compared with the other two groups (mut-/exp- and mut-/exp+) (1 vs 3 and 2 vs 3). These findings suggest that the TERT promoter mutation confers aggressive properties to PTCs.

RFS was evaluated using the Kaplan-Meier method and Cox proportional hazard models. In this analysis, we excluded four cases that had distant metastasis at the time of operation and additional 20 cases that were followed for less than six months. The survival curves 
of the three groups separated, and there was a statistically significant trend (Fig. 3A; log-rank trend, $\mathrm{p}<0.001)$. The HRs for recurrence of the mut+/exp+ group relative to the mut-/exp- group and the mut-/exp+ group after adjustment for age, sex, tumor size, extrathyroidal extension, and lymph node metastasis were 20.47 (95\% CI: 4.54 to 114.1, $\mathrm{p}<0.001)$ and 5.38 (95\% CI: 1.14 to $30.32, \mathrm{p}=0.046)$, respectively (Table 2 , 1 st and 2 nd comparisons). In the optimal models, the HRs of the mut+/exp+ group relative to the mut/exp- group and the mut-/exp+ group were 23.39 (95\% CI: 4.49 to 121.85 , p<0.001) and 6.24 (95\% CI: 1.44 to $27.13, \mathrm{p}=0.015$ ), respectively (Table 2, 1st and 2nd comparisons).

In two-group analysis, based on the mutational status (mut-/exp- and mut-/exp+ vs mut $+/ \exp ^{+}$) and the expression status (mut-/exp- vs mut-/exp+ and mut+/exp+), the Kaplan-Meier curves and the HRs are shown in Supplementary Fig. S2A, B and Supplementary Table 2, respectively.

Regarding the grouping based on the expression status (regardless of the mutational status), we attempted to set a threshold based on the concept that minimal expression could have a negligible effect on the clinical behavior because many of the mut-/exp+ tumors showed very low expression compared with the mut+/exp+ group (Fig. 2C). First, we calculated HRs in serial optimal Cox models for each cut-off percentile (mRNA expression-negative plus expression levels below each percentile cases vs expression levels above the percentile cases) (Supplementary Fig. S3A). We then ran Kaplan-Meier analyses between the 50th and 70th percentiles showing high HRs (Supplementary Fig. S3B). This allowed us to identify the optimal threshold, the 65th percentile (Supplementary Fig. S3B). Based on this, the HR adjusted for age, sex, tumor size, extrathyroidal extension, and lymph node metastasis was 4.12 (95\% CI: 1.55 to 10.72 , $\mathrm{p}=0.005)$. In the optimal model, the HR was 4.44 (95\% CI: 1.71 to $11.53, \mathrm{p}=0.002)$ (Supplementary Table S2, 3rd comparison). Other results using different adjustments are 
listed in Supplementary Table S2.

Relationship between TERT expression and clinicopathological features in the mutation-negative cases

376 Next, we focused on the TERT promoter mutation-negative cases. Interestingly, there were no statistical differences between the mut-/exp- group and the mut-/exp+ group in all clinicopathological parameters except mean recurrence time (Table 1, 1 vs 2). The Kaplan-Meier curve showed a significant difference (Fig. 3B, p=0.046), and Cox proportional hazard models also demonstrated statistical significance after adjustment for covariates (Table 2, 3rd comparison). The HR of the mut-/exp+ group relative to the mutlexp- group adjusted for age, sex, tumor size, extrathyroidal extension, and lymph node metastasis was 4.25 (95\% CI: 1.15 to $17.71, \mathrm{p}=0.041)$ (Table 2, 3rd comparison). In the optimal model, the HR was 4.24 (95\% CI: 1.13 to $15.90, \mathrm{p}=0.032$ ) (Table 2, 3rd comparison). Since the unadjusted HR was not significant $(p=0.067)$, we analyzed the relationship between age and the amount of TERT expression. Surprisingly, TERT expression was inversely correlated with age (Fig. 2D, $\mathrm{p}=0.002$ ), a finding that is opposite to the presence of TERT promoter mutations. These results indicate that TERT expression, even without presence of a TERT promoter mutation, has a negative influence on PTC prognosis.

Because many cases of the mut-/exp+ tumors showed very low TERT expression compared to the mut+/exp+ group (Fig. 2C), we also attempted to reclassify all mut- cases into two new groups based on the TERT expression level using the same method described above (Supplementary Fig. S3C). Kaplan-Meier analyses were run between the 75 th and 85th percentiles, and the optimal threshold was determined to be the 80th percentile (Supplementary Fig. S3D). Using this threshold, the unadjusted HR was 5.72 (95\% CI: 
calculated based on the presence or absence of the expression (exp- vs exp+). In the optimal model, the HR was 4.34 (95\% CI: 1.11 to 16.94, p=0.035) (Table 2, 4th comparison).

Using this grouping, there were significant differences in age (the expression levels above the 80th percentile group was younger), stage, recurrence, recurrence time, the prevalence of the BRAF mutation (Table 3). Interestingly, all cases with an expression level above the 80th percentile were stage I and displayed a significantly lower rate of the $B R A F$ mutation (30.0\% vs 69.9\%) (Table 3). Even though tumors in which the expression levels were above the 80th percentile were present in patients with younger age, lower stage, and a lower BRAF mutation rate, their prognosis was worse. The Kaplan-Meier curve also demonstrated a significant difference (Fig. $3 \mathrm{C}, \mathrm{p}=0.007$ ).

\section{DISSCUSSION}

First, in the present study, we have successfully reconfirmed the strong correlation between the presence of TERT promoter mutations and aggressive clinicopathological features in this PTC series. Second, we have demonstrated that there is a subset of PTCs that express TERT mRNA even in the absence of the TERT promoter mutation (mut/exp+). In this cases, TERT expression conferred a significant negative impact on PTC prognosis, which was, however, not as high as in the cases with a TERT promoter mutation. Since TERT promoter mutations are only observed in elderly patients, TERT expression may be a promising marker in younger patients.

Among TERT mut-/exp+ cases (determined by regular sequencing), there seems to be a small number of tumors with low allelic frequency of the TERT promoter mutation. Hence, in addition to a low allelic frequency of TERT promoter mutations, there are other 
mechanisms that upregulate TERT expression in the majority of the TERT mut-/exp+ cases. In other types of cancers, it has been reported that amplification, structural variants, alternative splicing, and promoter methylation also upregulate TERT expression (37-44). Of note, the allelic frequency depends on tumor cell purity and sensitivity of a detection method. It is still unclear whether the low allelic frequency of the TERT promoter mutation has a significant influence on tumor aggressivity because the number of such cases in the current series was too small. Further studies are needed to fully understand the mechanisms of the TERT mRNA upregulation in PTCs.

In the current series, all of the TERT promoter mutation-positive cases were 55 years of age or older, and among the eight recurrent cases in this age group, seven had a TERT promoter mutation. Thus, the presence of a TERT promoter mutation is probably a good marker in elderly patients. In contrast, since there was no TERT promoter mutationpositive case in the patients younger than 55 years, this mutation is not useful in younger patients presenting with PTC. However, in these patients, TERT expression appears to have prognostic value.

In the TERT mut-/exp+ group, there were many tumors with a very low amount of TERT expression compared to the levels of TERT expression in the TERT promoter mutationpositive cases. Such a minimal expression may not have clinical significance; therefore, we attempted to seek the best threshold based on the TERT expression levels. In the present study, the highest HR was obtained when the mutation-negative cases were categorized into two groups: cases with expression levels above the 80th percentile and others. However, it is necessary to analyze a much larger number of cases to determine a proper threshold. In addition, the threshold may be influenced by the detection method. This is a limitation of the current study. Moreover, since an active surveillance management approach has been used for low-risk micro-PTCs in Japan, current cases 
may not be fully representative of the whole PTC spectrum. It should rather be considered as a proof of principle that high TERT expression is associated with risk for recurrence.

The TERT expression levels above the 80th percentile cases were associated with larger tumor size, more frequent lymph node metastasis and extrathyroidal extension, and more T3 and T4 tumors compared to those in the other group, yet these differences were not statistically significant (Table 3), which could be due to the very small number of cases (only 10) in the group. For the same reason, it was difficult to compare these cases with the mutation-positive ones. Intriguingly, in the mutation-negative cases, TERT expression levels were inversely correlated with patient age and the frequency of the BRAF mutation. We have reported that tumor size is inversely correlated with the presence of the BRAF mutation in pediatric and adolescent PTCs (45). Taken together, tumors without the BRAF mutation may likely have higher TERT expression levels and more aggressive features in young PTC patients.

In summary, as reported by others, we confirm the association between presence of TERT promoter mutations and aggressive clinicopathological characteristics in PTCs. Moreover, high TERT expression levels were observed in PTCs even in TERT promoter mutationnegative tumors in patients of all ages, and TERT expression was associated with worse prognosis. Since TERT promoter mutations are only found in elderly patients, TERT expression can be also a useful marker, especially in younger patients with PTC.

\section{ACKNOWLEDGMENTS}

This study was supported in part by Grants-in-Aid for Scientific Research (JSPS KAKENHI) Grant Numbers 26293222, 26293142, and 16K09804. 


\section{DISCLOSURE STATEMENT}

The authors have nothing to disclose.

Correspondence and reprint requests: Norisato Mitsutake, $\mathrm{MD}, \mathrm{PhD}$.

Department of Radiation Medical Sciences, Atomic Bomb Disease Institute, Nagasaki University, Nagasaki, Japan.

Tel: +81-95-819-7116

E-mail: $\underline{\text { mitsu@nagasaki-u.ac.jp }}$

492

\section{REFERENCES}

1. Wiltshire JJ, Drake TM, Uttley L, Balasubramanian SP 2016 Systematic Review

2. Schlumberger MJ 1998 Papillary and follicular thyroid carcinoma. N Engl J Med of Trends in the Incidence Rates of Thyroid Cancer. Thyroid 26:1541-1552.

3. Ito Y, Miyauchi A, Kihara M, Fukushima M, Higashiyama T, Miya A 2018 Overall Survival of Papillary Thyroid Carcinoma Patients: A Single-Institution LongTerm Follow-Up of 5897 Patients. World J Surg 42:615-622.

4. Xing M 2005 BRAF mutation in thyroid cancer. Endocr Relat Cancer 12:245-262.

5. Matsuse M, Yabuta T, Saenko V, Hirokawa M, Nishihara E, Suzuki K, Yamashita S, Miyauchi A, Mitsutake N 2017 TERT promoter mutations and Ki-67 labeling index as a prognostic marker of papillary thyroid carcinomas: combination of two independent factors. Sci Rep 7:41752. 
507 6. Landa I, Ganly I, Chan TA, Mitsutake N, Matsuse M, Ibrahimpasic T, Ghossein RA, Fagin JA 2013 Frequent somatic TERT promoter mutations in thyroid cancer: higher prevalence in advanced forms of the disease. J Clin Endocrinol Metab 98:E1562-1566.

7. Liu X, Bishop J, Shan Y, Pai S, Liu D, Murugan AK, Sun H, El-Naggar AK, Xing M 2013 Highly prevalent TERT promoter mutations in aggressive thyroid cancers. Endocr Relat Cancer 20:603-610.

8. Alzahrani AS, Alsaadi R, Murugan AK, Sadiq BB 2016 TERT Promoter

10. Huang FW, Hodis E, Xu MJ, Kryukov GV, Chin L, Garraway LA 2013 Highly recurrent TERT promoter mutations in human melanoma. Science 339:957-959.

11. Horn S, Figl A, Rachakonda PS, Fischer C, Sucker A, Gast A, Kadel S, Moll I, Nagore E, Hemminki K, Schadendorf D, Kumar R 2013 TERT promoter mutations in familial and sporadic melanoma. Science 339:959-961.

12. Koh CM, Khattar E, Leow SC, Liu CY, Muller J, Ang WX, Li Y, Franzoso G, Li S, Guccione E, Tergaonkar V 2015 Telomerase regulates MYC-driven oncogenesis independent of its reverse transcriptase activity. J Clin Invest 125:2109-2122.

13. Low KC, Tergaonkar V 2013 Telomerase: central regulator of all of the hallmarks of cancer. Trends Biochem Sci 38:426-434.

14. Li Y, Tergaonkar V 2014 Noncanonical functions of telomerase: implications in telomerase-targeted cancer therapies. Cancer Res 74:1639-1644. 
15. Xing M, Liu R, Liu X, Murugan AK, Zhu G, Zeiger MA, Pai S, Bishop J 2014 BRAF V600E and TERT promoter mutations cooperatively identify the most aggressive papillary thyroid cancer with highest recurrence. J Clin Oncol 32:2718-2726.

16. Qasem E, Murugan AK, Al-Hindi H, Xing M, Almohanna M, Alswailem M, Alzahrani AS 2015 TERT promoter mutations in thyroid cancer: a report from a Middle Eastern population. Endocr Relat Cancer 22:901-908.

17. George JR, Henderson YC, Williams MD, Roberts DB, Hei H, Lai SY, Clayman GL 2015 Association of TERT Promoter Mutation, But Not BRAF Mutation, With Increased Mortality in PTC. J Clin Endocrinol Metab 100:E1550-1559.

18. Bullock M, Ren Y, O'Neill C, Gill A, Aniss A, Sywak M, Sidhu S, Delbridge L, Learoyd D, de Vathaire F, Robinson BG, Clifton-Bligh RJ 2016 TERT promoter mutations are a major indicator of recurrence and death due to papillary thyroid carcinomas. Clin Endocrinol (Oxf) 85:283-290.

19. Lee SE, Hwang TS, Choi YL, Han HS, Kim WS, Jang MH, Kim SK, Yang JH 2016 Prognostic Significance of TERT Promoter Mutations in Papillary Thyroid Carcinomas in a BRAF(V600E) Mutation-Prevalent Population. Thyroid 26:901910.

20. Bu R, Siraj AK, Divya SP, Kong Y, Parvathareddy SK, Al-Rasheed M, Al-Obaisi KAS, Victoria IG, Al-Sobhi SS, Al-Dawish M, Al-Dayel F, Al-Kuraya KS 2018 Telomerase reverse transcriptase mutations are independent predictor of diseasefree survival in Middle Eastern papillary thyroid cancer. Int J Cancer 142:20282039.

21. Melo M, da Rocha AG, Vinagre J, Batista R, Peixoto J, Tavares C, Celestino R, Almeida A, Salgado C, Eloy C, Castro P, Prazeres H, Lima J, Amaro T, Lobo C, Martins MJ, Moura M, Cavaco B, Leite V, Cameselle-Teijeiro JM, Carrilho F, Carvalheiro M, Maximo V, Sobrinho-Simoes M, Soares P 2014 TERT promoter 
mutations are a major indicator of poor outcome in differentiated thyroid carcinomas. J Clin Endocrinol Metab 99:E754-765.

22. Liu X, Qu S, Liu R, Sheng C, Shi X, Zhu G, Murugan AK, Guan H, Yu H, Wang

28. Oishi N, Kondo T, Ebina A, Sato Y, Akaishi J, Hino R, Yamamoto N, Mochizuki 

alterations of coexisting thyroid papillary carcinoma and anaplastic carcinoma: identification of TERT mutation as an independent risk factor for transformation. Mod Pathol 30:1527-1537.

29. Paulsson JO, Mu N, Shabo I, Wang N, Zedenius J, Larsson C, Juhlin CC 2018

32. 2017 AJCC Cancer Staging Manual, 8th edition. Springer International Publishing, New York.

33. Tuttle RM, Haugen B, Perrier ND 2017 Updated American Joint Committee on Cancer/Tumor-Node-Metastasis Staging System for Differentiated and Anaplastic Thyroid Cancer (Eighth Edition): What Changed and Why? Thyroid 27:751-756.

34. Ghossein RA, Katabi N, Fagin JA 2013 Immunohistochemical detection of mutated BRAF V600E supports the clonal origin of BRAF-induced thyroid cancers along the spectrum of disease progression. J Clin Endocrinol Metab 98:E1414-1421.

35. Nikiforova MN, Wald AI, Roy S, Durso MB, Nikiforov YE 2013 Targeted nextgeneration sequencing panel (ThyroSeq) for detection of mutations in thyroid 
cancer. J Clin Endocrinol Metab 98:E1852-1860.

36. Guerra A, Fugazzola L, Marotta V, Cirillo M, Rossi S, Cirello V, Forno I, Moccia T, Budillon A, Vitale M 2012 A high percentage of BRAFV600E alleles in papillary thyroid carcinoma predicts a poorer outcome. J Clin Endocrinol Metab 97:2333-2340.

37. Barthel FP, Wei W, Tang M, Martinez-Ledesma E, Hu X, Amin SB, Akdemir KC, Seth S, Song X, Wang Q, Lichtenberg T, Hu J, Zhang J, Zheng S, Verhaak RG 2017 Systematic analysis of telomere length and somatic alterations in 31 cancer types. Nat Genet 49:349-357.

38. Castelo-Branco P, Choufani S, Mack S, Gallagher D, Zhang C, Lipman T, Zhukova N, Walker EJ, Martin D, Merino D, Wasserman JD, Elizabeth C, Alon N, Zhang L, Hovestadt V, Kool M, Jones DT, Zadeh G, Croul S, Hawkins C, Hitzler J, Wang JC, Baruchel S, Dirks PB, Malkin D, Pfister S, Taylor MD, Weksberg R, Tabori U 2013 Methylation of the TERT promoter and risk stratification of childhood brain tumours: an integrative genomic and molecular study. Lancet Oncol 14:534-542.

39. Fan Y, Lee S, Wu G, Easton J, Yergeau D, Dummer R, Vogel P, Kirkwood JM, Barnhill RL, Pappo A, Bahrami A 2016 Telomerase Expression by Aberrant Methylation of the TERT Promoter in Melanoma Arising in Giant Congenital Nevi. J Invest Dermatol 136:339-342.

40. Seynnaeve B, Lee S, Borah S, Park Y, Pappo A, Kirkwood JM, Bahrami A 2017 Genetic and Epigenetic Alterations of TERT Are Associated with Inferior Outcome in Adolescent and Young Adult Patients with Melanoma. Sci Rep 7:45704.

41. Peifer M, Hertwig F, Roels F, Dreidax D, Gartlgruber M, Menon R, Kramer A, Roncaioli JL, Sand F, Heuckmann JM, Ikram F, Schmidt R, Ackermann S, Engesser A, Kahlert Y, Vogel W, Altmuller J, Nurnberg P, Thierry-Mieg J, Thierry- 
Mieg D, Mariappan A, Heynck S, Mariotti E, Henrich KO, Gloeckner C, Bosco G, Leuschner I, Schweiger MR, Savelyeva L, Watkins SC, Shao C, Bell E, Hofer T, Achter V, Lang U, Theissen J, Volland R, Saadati M, Eggert A, de Wilde B, 
672 Figure 1. Low allelic frequencies of the TERT promoter mutation in three samples, PTC A, PTC B, and PTC C. (A) Droplet digital PCR results. Each dot represents a positive droplet of a mutant allele or a wild-type allele. NTC: non-template control, Wt: wild-type control, hetMut: both mutant and wild-type control (monoallelic). (B) Sanger sequencing chromatograms of indicated samples. The hot spots of the TERT promoter mutation are shown as arrowheads. All of PTC A, PTC B, and PTC C had the C228T mutation.

Figure 2. Summary of the TERT mutational and expression status in the current series. (A) The flowchart of the TERT mutation/expression screening. (B) Pie chart of the results. (C) Relative TERT expression level in each group classified using the above status. (D) Correlation between the TERT expression level and patient age. The mutationnegative/expression-positive cases are plotted. Solid line represents the linear regression model with $95 \%$ confidence intervals indicated by dotted lines. Arrow heads indicate cases with TERT mRNA expression higher than the 80th percentile shown in C.

686

Figure 3. Kaplan-Meier curves of recurrence-free survival. The vertical tick marks correspond to censored data. (A) The recurrence-free survival curves of the indicated groups. p-value of a log-rank trend test is shown. (B) and (C) Only mutation-negative cases were analyzed. p-values of log-rank tests are shown.

691

692 
Table 1. Association between TERT mutational/expression status and clinicopathological features

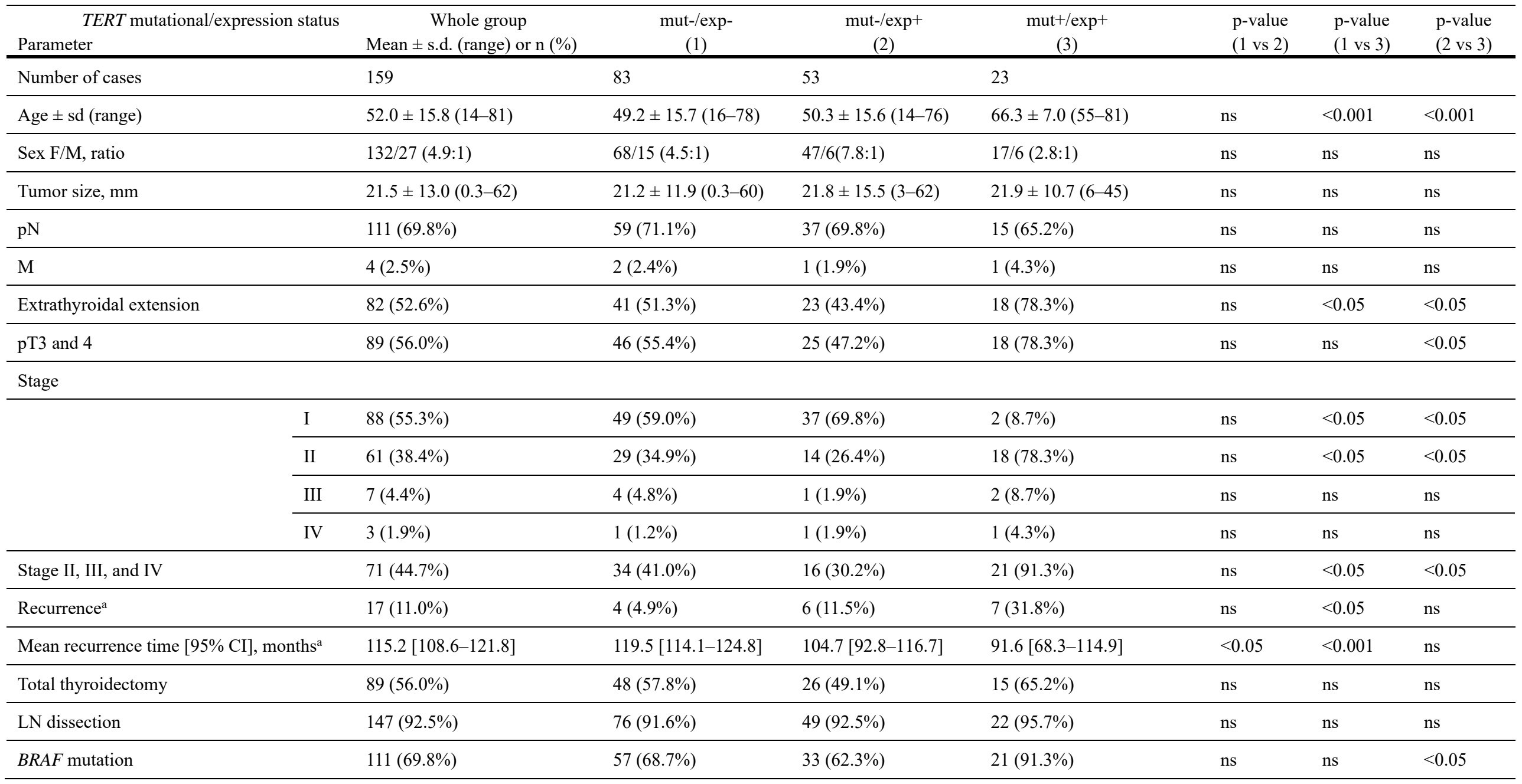

ns: not significant, $\mathrm{p} \geq 0.05$

mut-/exp-: TERT promoter mutation-negative/mRNA expression-negative

mut-/exp+: TERT promoter mutation-negative/mRNA expression-positive

mut+/exp+: TERT promoter mutation-positive/mRNA expression-positive

${ }^{\text {a }}$ our cases with distant metastasis and 20 cases that were followed for less than six months were not included. 
Table 2. Hazard ratios of disease recurrence

\begin{tabular}{|c|c|c|c|c|c|c|c|c|c|c|c|c|}
\hline & HR & $95 \% \mathrm{CI}$ & p-value & HR & $95 \% \mathrm{CI}$ & p-value & HR & $95 \% \mathrm{CI}$ & p-value & HR & $95 \% \mathrm{CI}$ & $\mathrm{p}$-value \\
\hline mut-/exp- & 1.00 & & & 1.00 & & & 1.00 & & & 1.00 & & \\
\hline mut $+/$ exp + & 7.53 & $2.39-26.69$ & 0.001 & 20.25 & $4.54-109.26$ & $<0.001$ & 20.47 & $4.54-114.1$ & $<0.001$ & 23.39 & $4.49-121.85$ & $<0.001$ \\
\hline adjustment: & & & & \multicolumn{3}{|c|}{ age, sex } & \multicolumn{3}{|c|}{ age, sex, size, Ex, N } & \multicolumn{3}{|c|}{ optimal model (age)* } \\
\hline mut-/exp+ & 1.00 & & & 1.00 & & & 1.00 & & & 1.00 & & \\
\hline mut $+/ \exp +$ & 2.34 & $0.80-6.99$ & 0.131 & 5.07 & $1.24-24.03$ & 0.034 & 5.38 & $1.14-30.32$ & 0.046 & 6.24 & $1.44-27.13$ & 0.015 \\
\hline adjustment: & & & & \multicolumn{3}{|c|}{ age, sex } & \multicolumn{3}{|c|}{ age, sex, size, Ex, N } & \multicolumn{3}{|c|}{ optimal model (age)* } \\
\hline mut-/exp- & 1.00 & & & 1.00 & & & 1.00 & & & 1.00 & & \\
\hline mut-/exp+ & 3.27 & $0.98-11.87$ & 0.067 & 4.65 & $1.31-18.51$ & 0.026 & 4.25 & $1.15-17.71$ & 0.041 & 4.24 & $1.13-15.90$ & 0.032 \\
\hline adjustment: & & & & \multicolumn{3}{|c|}{ age, sex } & \multicolumn{3}{|c|}{ age, sex, size, Ex, $\mathrm{N}$} & \multicolumn{3}{|c|}{ optimal model (age)* } \\
\hline mut-/exp- and exp below $80^{\text {th }}$ percentile & 1.00 & & & 1.00 & & & 1.00 & & & 1.00 & & \\
\hline mut-/exp above $80^{\text {th }}$ percentile & 5.72 & $1.39-19.23$ & 0.010 & 5.09 & $1.21-17.81$ & 0.022 & 3.36 & $0.80-11.75$ & 0.095 & 4.34 & $1.11-16.94$ & 0.035 \\
\hline adjustment: & & & & & age, sex & & & age, sex, size, I & & & timal model (a & \\
\hline
\end{tabular}

*In the optimal model, only "age" was used for adjustment.

mut-/exp-: TERT promoter mutation-negative/mRNA expression-negative

mut-/exp+: TERT promoter mutation-negative/mRNA expression-positive

mut+/exp+: TERT promoter mutation-positive/mRNA expression-positive

mut-/exp- and exp below $80^{\text {th }}$ percentile: TERT promoter mutation-negative/mRNA expression-negative and mRNA expression levels below the 80th percentile of the mut-/exp+ cases

mut-/exp above $80^{\text {th }}$ percentile: TERT mRNA expression levels above the 80th percentile of the mut-/exp+ cases 
Table 3. Association between TERT expression and clinicopathological features in the TERT promoter mutation-negative cases

\begin{tabular}{|c|c|c|c|c|}
\hline TERT expression & $\begin{array}{l}\text { Whole group } \\
\text { Mean } \pm \text { s.d. (range) or } n(\%)\end{array}$ & $\begin{array}{l}\text { TERT exp- and exp } \\
\text { below } 80^{\text {th }} \text { percentile } \\
\end{array}$ & $\begin{array}{l}\text { TERT exp above } \\
80^{\text {th }} \text { percentile } \\
\end{array}$ & $\begin{array}{l}\text { Univariate } \\
\text { p-value }\end{array}$ \\
\hline Number of cases & 136 & 126 & 10 & \\
\hline Age & $49.6 \pm 15.6(14-78)$ & $50.5 \pm 15.6(14-78)$ & $38.8 \pm 12.6(20-52)$ & $<0.05$ \\
\hline Sex F/M, ratio & $115 / 21(5.5: 1)$ & $106 / 20(5.3: 1)$ & 9/1 (9.0:1) & ns \\
\hline Tumor size, mm & $21.4 \pm 13.4(0.3-62)$ & $21.1 \pm 13.1(0.3-62)$ & $24.7 \pm 16.9(4-55)$ & ns \\
\hline $\mathrm{pN}$ & $96(70.6 \%)$ & $88(69.8 \%)$ & $8(80.0 \%)$ & ns \\
\hline M & $3(2.2 \%)$ & $3(2.4 \%)$ & 0 & ns \\
\hline Extrathyroidal extension & $64(48.1 \%)$ & $58(47.2 \%)$ & $6(60.0 \%)$ & ns \\
\hline pT3 and 4 & $71(52.2 \%)$ & $65(51.6 \%)$ & $6(60.0 \%)$ & ns \\
\hline \multicolumn{5}{|l|}{ Stage } \\
\hline I & $86(63.2 \%)$ & $76(60.3 \%)$ & $10(100.0 \%)$ & $<0.05$ \\
\hline II & $43(31.6 \%)$ & $43(34.1 \%)$ & 0 & $<0.05$ \\
\hline III & $5(3.7 \%)$ & $5(4.0 \%)$ & 0 & ns \\
\hline IV & $2(1.5 \%)$ & $2(1.6 \%)$ & 0 & ns \\
\hline Stage II, III and IV & $50(36.8 \%)$ & $50(39.7 \%)$ & 0 & $<0.05$ \\
\hline Recurrence $^{\mathrm{a}}$ & $10(7.5 \%)$ & $7(5.7 \%)$ & $3(30.0 \%)$ & $<0.05$ \\
\hline Mean recurrence time $[95 \% \mathrm{CI}]$, months ${ }^{\mathrm{a}}$ & $115.2[109.4-121.0]$ & $117.6[112.1-123.0]$ & $86.3[53.9-118.7]$ & $<0.05$ \\
\hline Total thyroidectomy & $74(54.4 \%)$ & $67(53.2 \%)$ & $7(70.0 \%)$ & ns \\
\hline LN dissection & $125(91.9 \%)$ & $116(92.1 \%)$ & $9(90.0 \%)$ & ns \\
\hline$B R A F$ mutation & 90 (66.2\%) & $87(69.0 \%)$ & $3(30.0 \%)$ & $<0.05$ \\
\hline
\end{tabular}

ns: not significant, $\mathrm{p} \geq 0.05$

athree cases with distant metastasis and 20 cases that were followed for less than six months were not included.

$T E R T$ exp- and exp below $80^{\text {th }}$ percentile: TERT mRNA expression-negative and mRNA expression levels below the 80th percentile

TERT exp- above $80^{\text {th }}$ percentile: TERT mRNA expression levels above the 80th percentile 
Figure 1

A

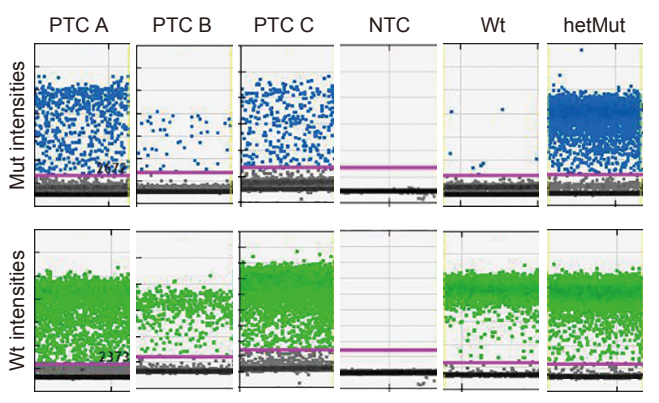

B 5 - TGGGAGGGCCCGGAGGGGCTGGCCGGGGACCCGGGAGGET -3 '

PTC A

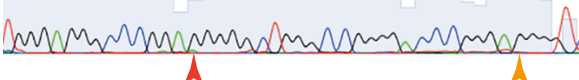

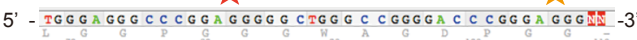

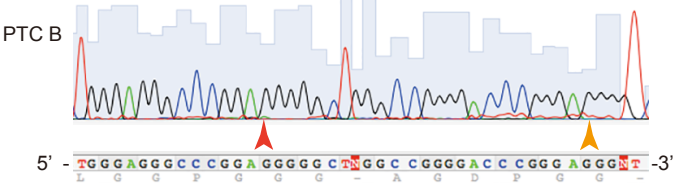

PTC C

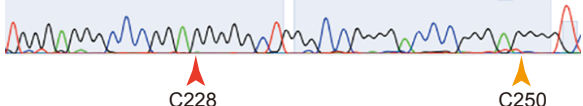


Figure 2

A

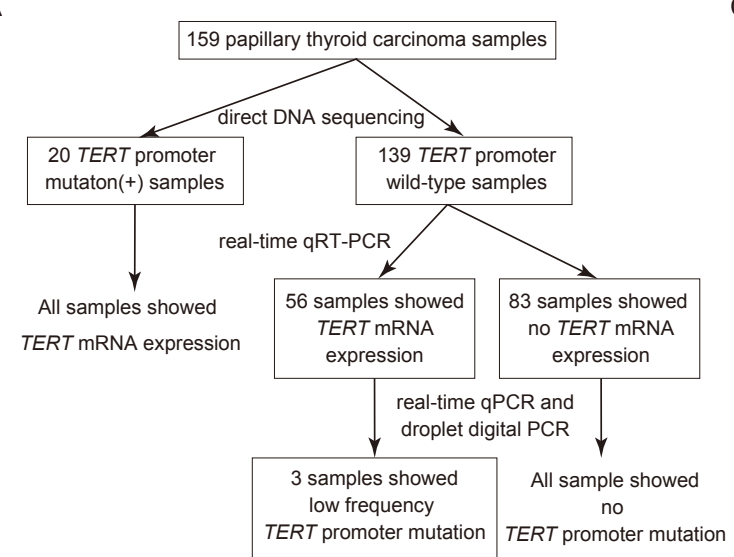

C

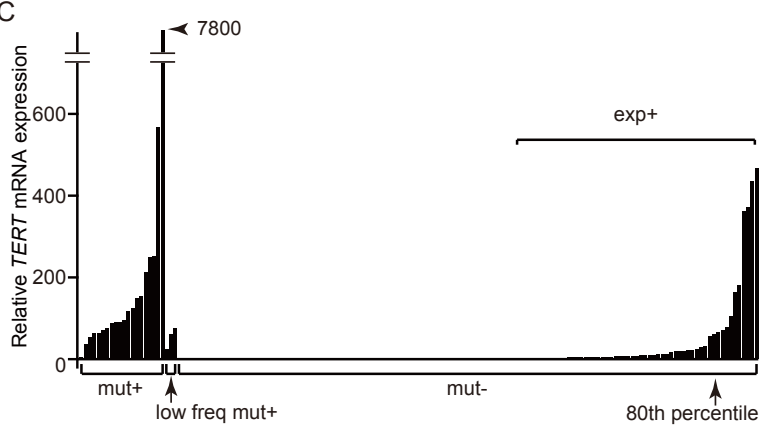

D

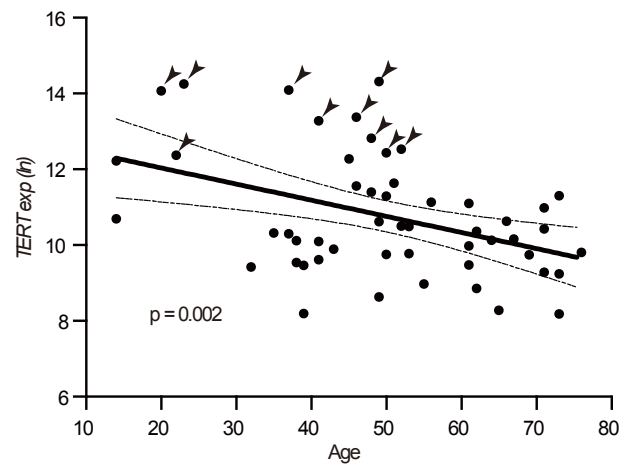

B

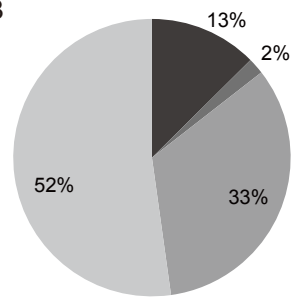

TERT mut+/exp+: 20 cases

TERT low freq mut+/exp+: 3 cases

TERT mut-/exp+: 53 cases

TERT mut-/exp-: 83 cases 
Figure 3

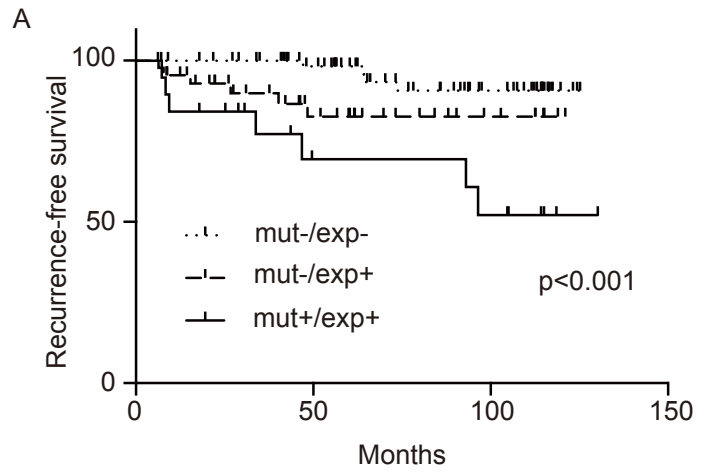

B

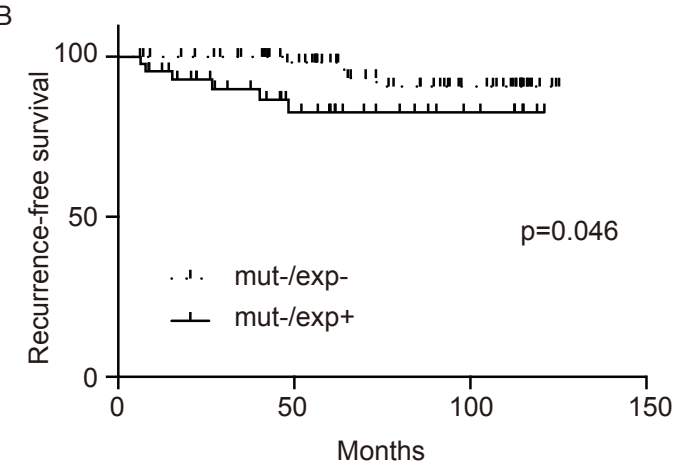

C

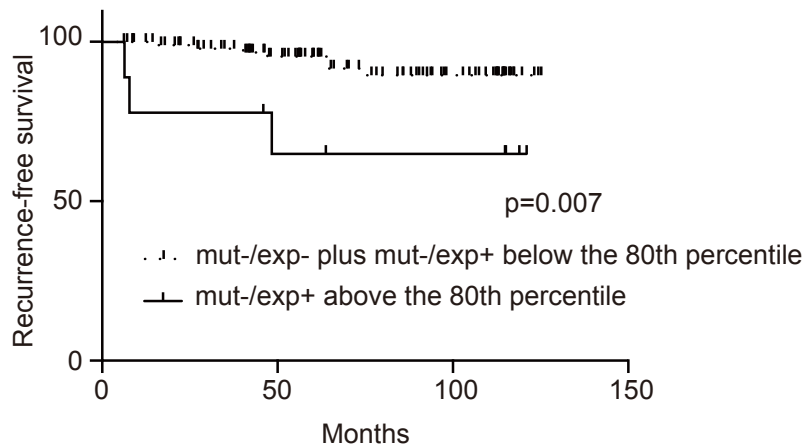


Supplementary Table 1. Allelic frequencies of the TERT promoter mutation in cancer cells.

\begin{tabular}{|c|c|c|c|c|c|c|c|}
\hline & \multicolumn{2}{|c|}{ Concentration (copies/ $\mu \mathrm{l})^{\mathrm{a}}$} & \multirow{2}{*}{$\begin{array}{l}\text { TERT mutant } \\
\text { frequency }^{\mathrm{b}}\end{array}$} & \multicolumn{2}{|c|}{ Concentration $(\text { copies } / \mu \mathrm{l})^{\mathrm{a}}$} & \multirow[t]{2}{*}{ TERT/BRAF } & \multirow{2}{*}{$\begin{array}{c}\text { Allelic } \\
\text { frequency in } \\
\text { cancer cells }\end{array}$} \\
\hline & TERT wt & TERT mut & & TERT mut & $B R A F$ mut & & \\
\hline PTC A & 289 & 58.9 & 0.17 & 46 & 164 & 0.28 & 0.14 \\
\hline PTC B & 46.2 & 4.9 & 0.1 & 5.4 & 67 & 0.08 & 0.04 \\
\hline PTC C & 743 & 42.7 & 0.05 & 27.7 & 483 & 0.06 & 0.03 \\
\hline
\end{tabular}

average of the multiple experiments

${ }^{b}$ mutant frequency = mutant droplet copy number/(mutant droplet copy number + wild-type droplet copy number)) 
Supplementary Table 2. Hazard ratios of disease recurrence.

\begin{tabular}{|c|c|c|c|c|c|c|c|c|c|c|c|c|c|c|c|c|c|c|}
\hline & $\mathrm{HR}$ & $95 \% \mathrm{Cl}$ & $\mathrm{p}$-value & HR & $95 \% \mathrm{Cl}$ & p-value & $\mathrm{HR}$ & $95 \% \mathrm{Cl}$ & $p$-value & $\mathrm{HR}$ & $95 \% \mathrm{Cl}$ & $p$-value & HR & $95 \% \mathrm{Cl}$ & $\mathrm{p}$-value & $\mathrm{HR}$ & $95 \% \mathrm{Cl}$ & $\mathrm{p}$-value \\
\hline mut-/exp- & 1 & & & 1 & & & 1 & & & 1 & & & 1 & & & 1 & & \\
\hline \multirow[t]{2}{*}{ mut+lexp +} & 7.53 & $2.39-26.69$ & 0.001 & 20.25 & \multirow{2}{*}{\multicolumn{2}{|c|}{$\begin{array}{c}4.54-109.26 \\
\text { age, sex }\end{array}$}} & 19.44 & $4.36-103.95$ & $<0.001$ & 14.91 & $3.42-78.73$ & 0.001 & 20.47 & $4.54-114.1$ & $<0.001$ & 23.39 & $4.49-121.85$ & $<0.001$ \\
\hline & & & & & & & & \multicolumn{2}{|l|}{ age, sex, size } & \multicolumn{3}{|c|}{ age, sex, size, Ex } & \multicolumn{3}{|c|}{ age, sex, size, Ex, N } & \multicolumn{3}{|c|}{ optimal model (age) $)^{\star}$} \\
\hline mut-lexp+ & 1 & & & 1 & & & 1 & & & 1 & & & 1 & & & 1 & & \\
\hline mut+lexp + & 2.34 & $0.80-6.99$ & 0.131 & 5.07 & $1.24-24.03$ & 0.034 & 4.75 & $1.17-22.23$ & 0.042 & 3.12 & $0.77-14.97$ & 0.14 & 5.38 & $1.14-30.32$ & 0.046 & 6.24 & $1.44-27.13$ & 0.015 \\
\hline \multicolumn{4}{|l|}{ adjustment: } & \multicolumn{3}{|c|}{ age, sex } & \multicolumn{3}{|c|}{ age, sex, size } & \multicolumn{3}{|c|}{ age, sex, size, Ex } & \multicolumn{3}{|c|}{ age, sex, size, Ex, $N$} & \multicolumn{3}{|c|}{ optimal model (age $)^{*}$} \\
\hline mut-/exp- & 1 & & & 1 & & & 1 & & & 1 & & & 1 & & & 1 & & \\
\hline \multirow[t]{2}{*}{ mutt/exp+ } & 4.50 & $1.63-14.85$ & 0.007 & 6.00 & $2.10-20.64$ & 0.002 & 6.08 & $2.11-21.03$ & 0.002 & 6.62 & $2.26-23.21$ & 0.002 & 6.41 & $2.26-22.03$ & 0.002 & 4.32 & $1.40-13.29$ & 0.011 \\
\hline & & & & \multicolumn{3}{|c|}{ age, sex } & \multicolumn{3}{|c|}{ age, sex, size } & \multicolumn{3}{|c|}{ age, sex, size, Ex } & \multicolumn{3}{|c|}{ age, sex, size, Ex, N } & \multicolumn{3}{|c|}{ optimal model $(\mathrm{Ex})^{*}$} \\
\hline mut-lexp \pm & 1 & & & 1 & & & 1 & & & 1 & & & 1 & & & 1 & & \\
\hline \multirow[t]{2}{*}{ mut+/exp+ } & 4.68 & $1.75-11.90$ & 0.002 & 11.14 & $2.95-49.55$ & 0.001 & 10.55 & $2.79-46.26$ & 0.001 & 8.01 & $2.12-35.59$ & 0.004 & 13.80 & $3.39-66.67$ & 0.001 & 11.83 & $2.84-49.37$ & 0.001 \\
\hline & & & & \multicolumn{3}{|c|}{ age, sex } & \multicolumn{3}{|c|}{ age, sex, size } & \multicolumn{3}{|c|}{ age, sex, size, Ex } & \multicolumn{3}{|c|}{ age, sex, size, Ex, $\mathrm{N}$} & \multicolumn{3}{|c|}{ optimal model (age) ${ }^{*}$} \\
\hline mutt/exp- and exp below 65 th percentile & 1 & & & 1 & & & 1 & & & 1 & & & \multicolumn{3}{|l|}{1} & 1 & & \\
\hline mut \pm /exp above 65 th percentile & 4.64 & $1.79-11.84$ & 0.002 & 5.2 & $1.94-13.69$ & 0.001 & 5.17 & $1.91-13.74$ & 0.001 & 4.42 & $1.65-11.69$ & 0.003 & 4.12 & $1.55-10.72$ & 0.005 & 4.44 & $1.71-11.53$ & 0.002 \\
\hline adjustment: & & & & & age, sex & & & age, sex, size & & & age, sex, size, E & & & sex, size, E & & & mal model (ac & \\
\hline mut-lexp- & 1 & & & 1 & & & 1 & & & 1 & & & 1 & & & 1 & & \\
\hline mut-lexp+ & 3.27 & $0.98-11.87$ & 0.067 & 4.65 & $1.31-18.51$ & 0.026 & 4.83 & $1.36-19.34$ & 0.024 & 5.21 & $1.46-21.14$ & 0.019 & 4.25 & $1.15-17.71$ & 0.041 & 4.24 & $1.13-15.9$ & 0.032 \\
\hline adjustment: & & & & & age, sex & & & age, sex, size & & & age, sex, size, Ex & & & sex, size, E. & & & mal model (as & \\
\hline mut-lexp- and exp below 80th percentile & 1 & & & 1 & & & 1 & & & 1 & & & 1 & & & 1 & & \\
\hline mut-lexp above 80th percentile & 5.72 & $1.39-19.23$ & 0.01 & 5.09 & $1.21-17.81$ & 0.022 & 5.43 & $1.29-18.88$ & 0.017 & 4.56 & $1.05-16.29$ & 0.037 & 3.36 & $0.8-11.75$ & 0.095 & 4.34 & $1.11-16.94$ & 0.035 \\
\hline adjustment: & & & & & age, sex & & & age, sex, size & & & age, sex, size, Ex & & & sex, size, E. & & & mal model (as & \\
\hline
\end{tabular}

* In the optimal model, only age was used for adjustment

mutt/exp- and exp below 65th percentile: TERT mRNA expression-negative and TERT mRNA expression levels below the 65 th percentile, regardless of the mutational status

mut \pm exp higher 35\%: TERT mRNA expression levels above the 65th percentile, regardless of the mutational status

mut-lexp- and exp below 80th percentile: TERT promoter mutation-negative/mRNA expression-negative and mRNA expression levels below the 80th percentile of the mut-lexp+ cases

mut-/exp above 80th percentile: TERT mRNA expression levels above the 80th percentile of the mut-lexp+ cases 
Supplementary Table 3. Association between TERT expression and clinicopathological features in all cases (including TERT promoter mutation-positive cases)

\begin{tabular}{|c|c|c|c|c|}
\hline TERT mutational status & $\begin{array}{c}\text { Whole group } \\
\text { Mean } \pm \text { s.d. (range) or } n(\%)\end{array}$ & $\begin{array}{l}\text { mut } \pm / \text { exp- and exp below } \\
\text { 65th percentile }\end{array}$ & $\begin{array}{l}\text { mut } \pm \text { /exp above } 65 \text { th } \\
\text { percentile }\end{array}$ & $\begin{array}{l}\text { Univariate } \\
\text { comparison }\end{array}$ \\
\hline Number of cases & 159 & 132 & 27 & \\
\hline Age & $52.0 \pm 15.8(14-81)$ & $51.2 \pm 15.6(14-78)$ & $56.0 \pm 16.4(20-81)$ & ns \\
\hline Sex F/M, ratio & $132 / 27(4.9: 1)$ & $112 / 20(5.6: 1)$ & $20 / 7(2.9: 1)$ & ns \\
\hline Tumor size, mm & $21.5 \pm 13.0(0.3-62)$ & $21.4 \pm 13.0(0.3-62)$ & $21.8 \pm 13.1(4-55)$ & ns \\
\hline $\mathrm{pN}$ & $111(69.8 \%)$ & $92(69.7 \%)$ & $19(70.4 \%)$ & ns \\
\hline M & $4(2.5 \%)$ & $3(2.3 \%)$ & $1(3.7 \%)$ & ns \\
\hline Extrathyroidal extension & $82(52.6 \%)$ & $63(48.8 \%)$ & $19(70.4 \%)$ & ns \\
\hline pT3 and 4 & $89(56.0 \%)$ & $70(53.0 \%)$ & $19(70.4 \%)$ & ns \\
\hline \multicolumn{5}{|l|}{ Stage } \\
\hline I & $88(55.3 \%)$ & $76(57.6 \%)$ & $12(44.4 \%)$ & ns \\
\hline II & $61(38.4 \%)$ & $48(36.4 \%)$ & $13(48.1 \%)$ & ns \\
\hline III & $7(4.4 \%)$ & $6(4.5 \%)$ & $1(3.7 \%)$ & ns \\
\hline IV & $3(1.9 \%)$ & $2(1.5 \%)$ & $1(3.7 \%)$ & ns \\
\hline Stage II, III and IV & $71(44.7 \%)$ & $56(42.4 \%)$ & $15(55.6 \%)$ & ns \\
\hline Recurrence $^{a}$ & $17(11.0 \%)$ & $9(7.0 \%)$ & $8(30.8 \%)$ & $<0.05$ \\
\hline Mean recurrence time $[95 \% \mathrm{Cl}]$, months $^{\mathrm{a}}$ & $115.2[108.6-121.8]$ & $116.1[110.4-121.7]$ & $91.6[69.9-113.4]$ & $<0.05$ \\
\hline Total thyroidectomy & $89(56.0 \%)$ & $72(54.5 \%)$ & $17(63.0 \%)$ & ns \\
\hline LN dissection & $147(92.5 \%)$ & $122(92.4 \%)$ & $25(92.6 \%)$ & ns \\
\hline$B R A F$ mutation & $111(69.8 \%)$ & 93 (70.5\%) & $18(66.7 \%)$ & ns \\
\hline
\end{tabular}

ns: not significant, $p \geq 0.05$

${ }^{a}$ four cases with distant metastases and 20 cases that were followed for less than six months were not included.

mut \pm /exp- and exp below 65th percentile: TERT mRNA expression-negative and TERT mRNA expression levels below the 65th percentile, regardless of the mutational status mut \pm /exp above 65th percentile: TERT mRNA expression levels above the 65th percentile, regardless of the mutational status 
Supplementary Figure S1
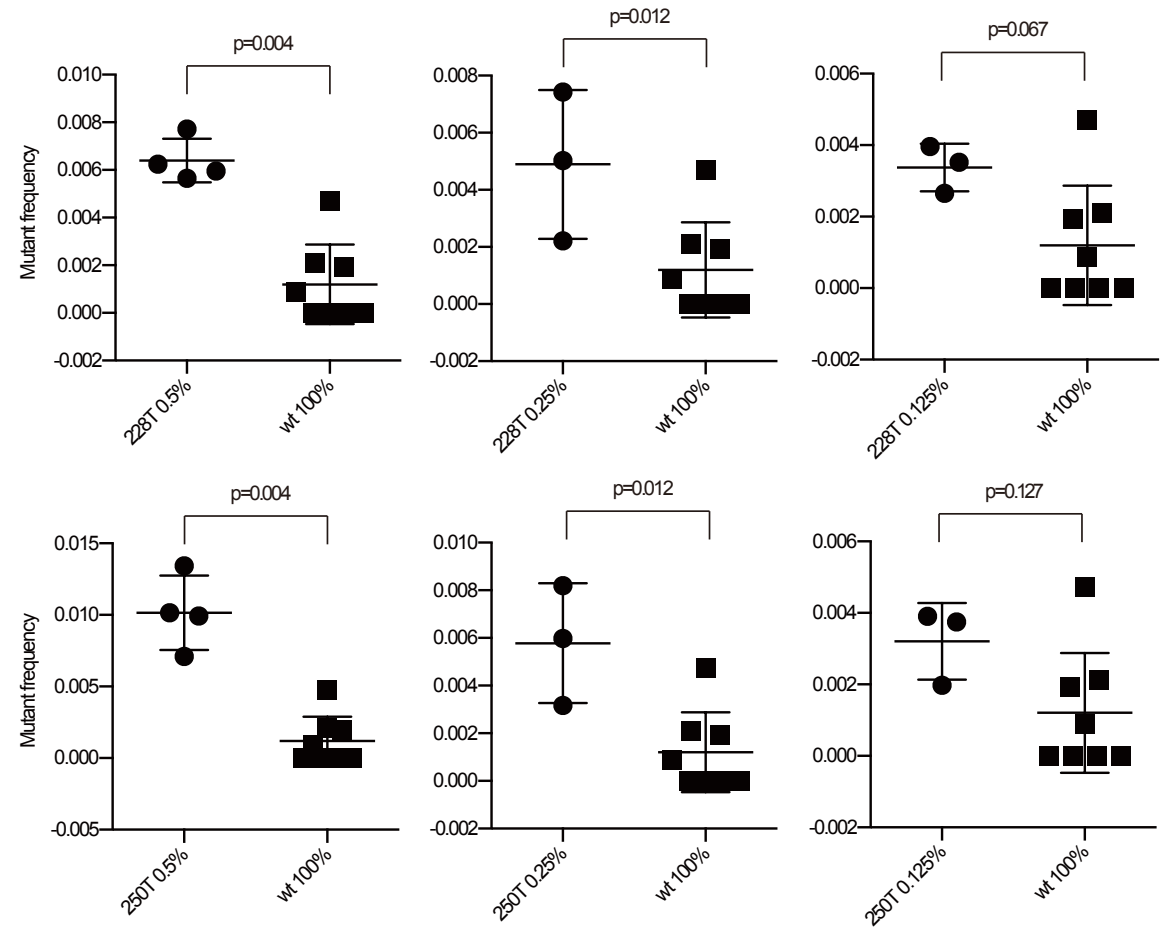

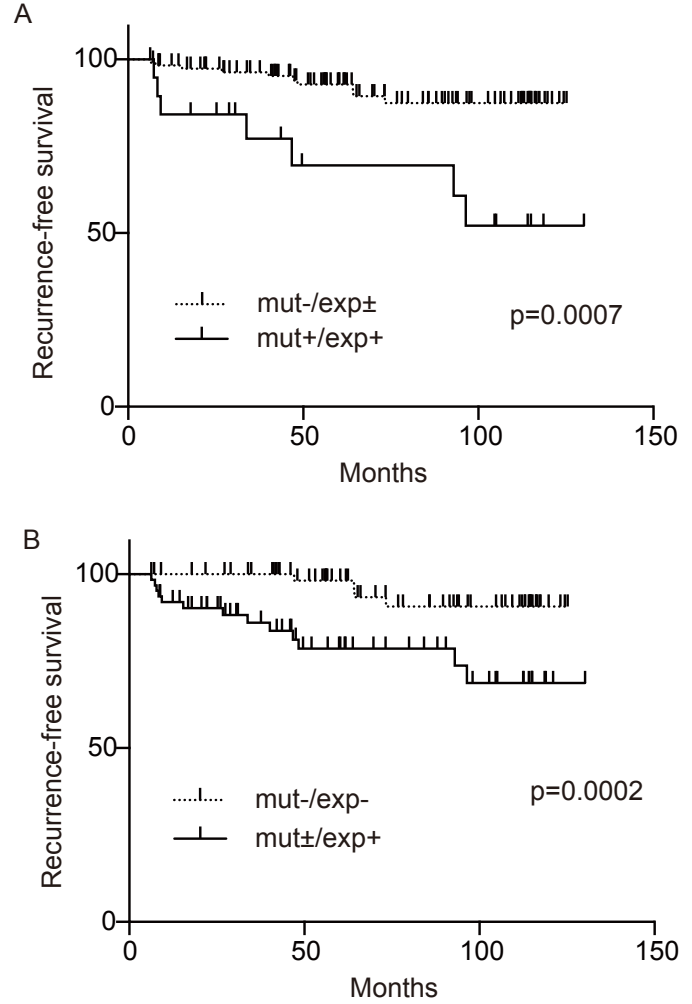
Supplementary Figure S3

A

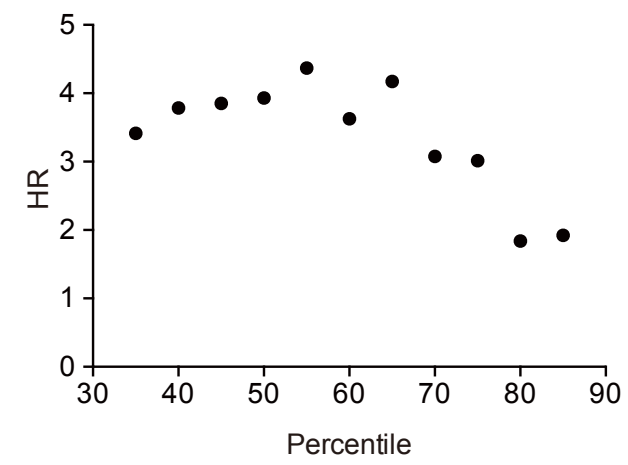

B
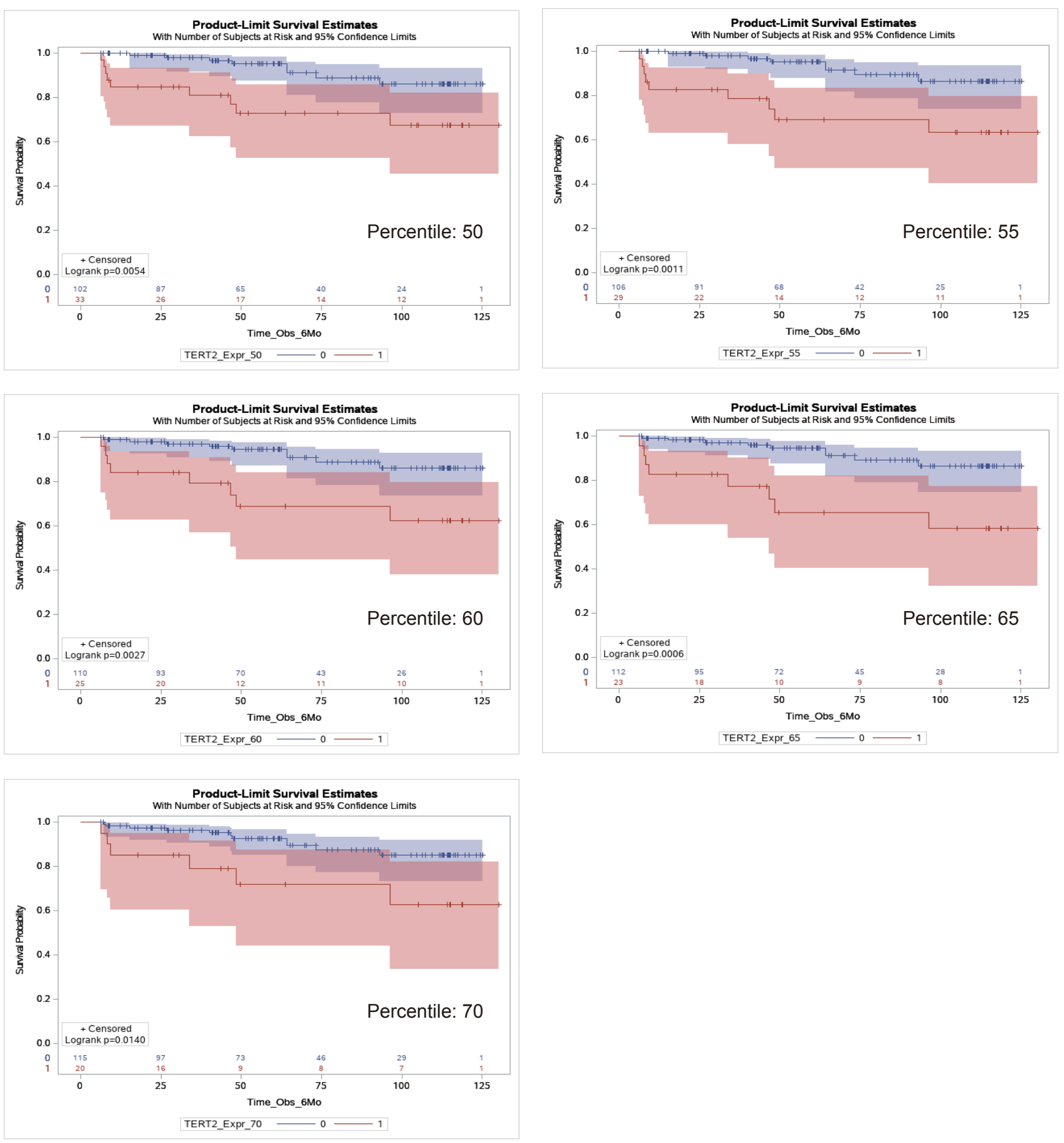
Supplementary Figure S3

C

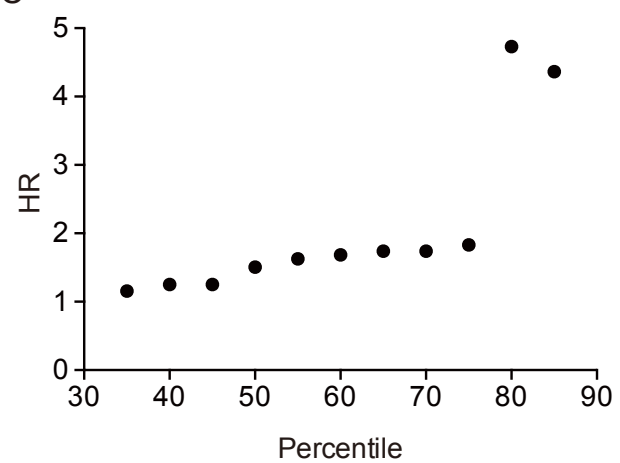

D
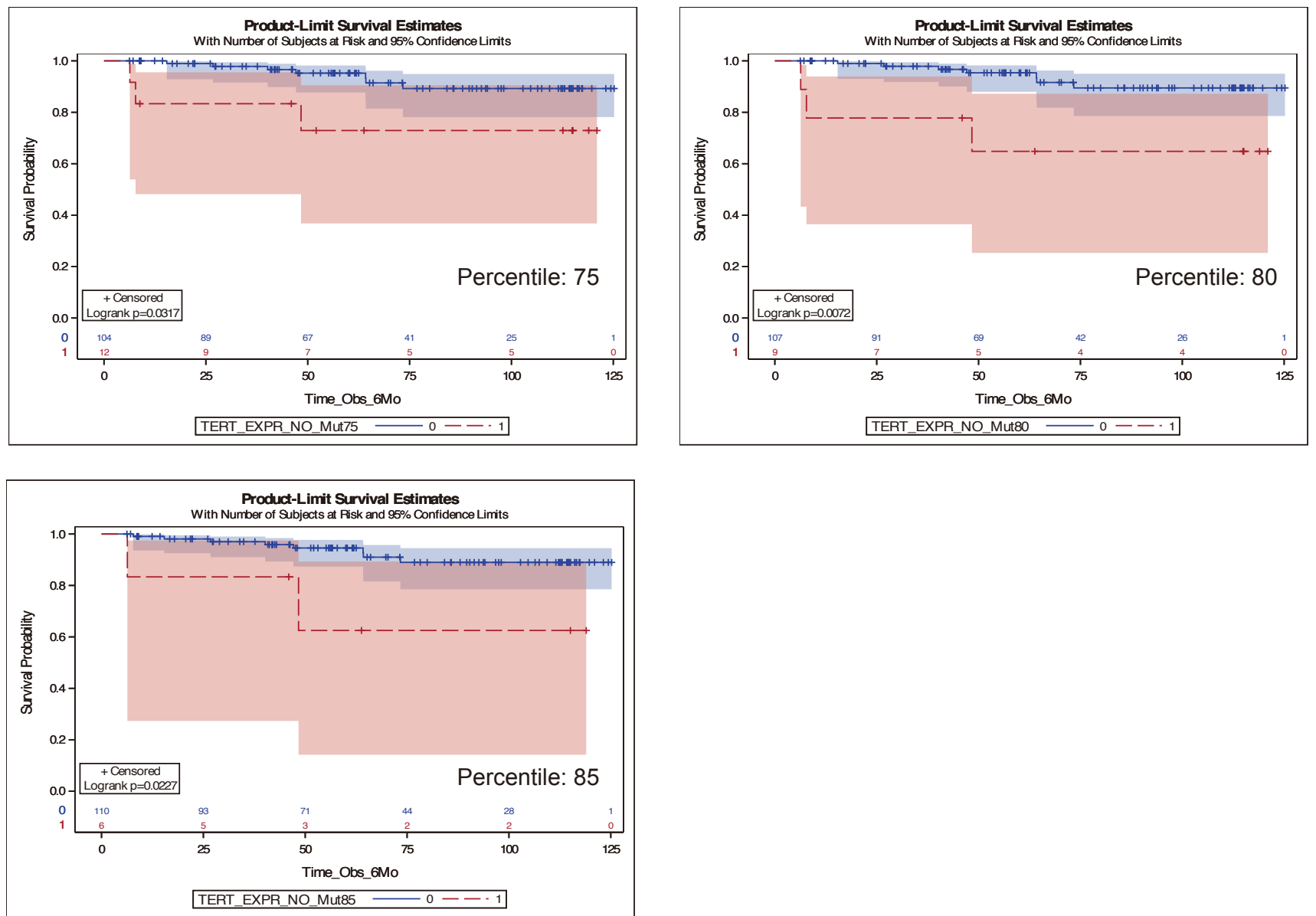


\section{SUPPLEMENTARY FIGURE LEGENDS}

\section{Figure S1. Detection limit of ddPCR.}

The detection limit of ddPCR was determined using serial dilutions of the PCR product of the TERT promoter mutation (C228T or C250T) in the wild-type PCR product. The TERT promoter region (163 bp) was amplified using DNA extracted from cell lines having only wild-type, C228T, and $\mathrm{C} 250 \mathrm{~T}$ and purified. We prepared a total 3,000 copies of the mixture of the mutant product $(0 \%, 0.125 \%, 0.25 \%$, and $0.5 \%)$ and the wild-type product and measured the mutant copy number using ddPCR. The results were compared with those of the $100 \%$ wild-type product, and the difference was analyzed by Mann-Whitney U test using the GraphPad Prism software. Significant difference was only observed between the $0.25 \%$ or more mutant allele samples (both C228T and $\mathrm{C} 250 \mathrm{~T})$ and the wild-type samples.

\section{Figure S2. Kaplan-Meier curves of the different grouping based on the TERT mutational/expression status.}

The vertical tick marks correspond to censored data. p-values of log-rank tests are shown. (A) The current cases were divided based on the mutational status. (B) The current cases were divided based on the expression status.

Figure S3. Hazard ratios and Kaplan-Meier curves of each cut-off percentile based on the TERT mRNA expression level.

(A) Hazard ratios were calculated including mutation-positive and negative cases. (B) KaplanMeier curves of the indicted cut-off percentiles (expression-negative plus expression levels below the indicated percentile $v s$ expression levels above the indicated percentile) (C) Hazard ratios were calculated using only mutation-negative cases. (D) Kaplan-Meier curves of the indicted cutoff percentiles (expression-negative plus expression levels below the indicated percentile $v s$ expression levels above the indicated percentile) 